\title{
VIRTUAL REALITY AND ITS IMPACT ON LEARNING SUCCESS
}

\author{
Thomas Keller, Elke Brucker-Kley and Christian Wyder \\ ZHAW School of Management and Law \\ Winterthur, Switzerland
}

\begin{abstract}
For elementary schools, the question arises as to whether the use of Virtual Reality (VR) can lead to better learning success for pupils. A field experiment was chosen as the methodology for investigating this question. A total of 82 pupils from four first and third secondary school classes were available for this field experiment. They were randomly assigned to a group per class, taking gender into account. Both groups attended a pre-test one week before the respective course unit in order to identify any previous knowledge. One week after the course unit, both groups again completed the post-test. The tests were designed in such a way that equal weight was attached to each learning objective of the learning units. During the evaluation, the individual learning success, i.e. the difference between the post-test and the pre-test, was calculated for each pupil. No significant differences in learning success between the traditional and the VR based units could be found. However, the VR learning unit showed a positive effect on the learning success and was widely accepted by the pupils. From an methodological point of view it is very difficult to design equivalent learning units and to compare them fairly.
\end{abstract}

\section{KEYWORDS}

Field Experiment, Learning Success, Virtual Reality

\section{INTRODUCTION}

Keller et al (2018) designed a learning unit on micro plastics supported by virtual reality technology. In this work he describes in detail the procedure from the motivation, the choice of a suitable learning content, the design and creation of the learning unit as well as an evaluation round with experts and students of a secondary school class in Switzerland. As essential artifacts, a procedure model for the creation of VR learning units as well as the VR learning unit on micro plastics emerged from his work. (Glauser \& Keller, 2018)

This paper is a continuation of Keller's work. However, the continuation does not concentrate on a revision of the VR course unit, but on a detailed evaluation of the influence of this VR course unit on the learning success of secondary school students. This continuation is useful because in (Keller et al., 2018) only an evaluation with a small sample have been made. In addition, the focus of his evaluation was on the learning unit itself and no systematic survey was carried out on the influence on learning success. (Glauser \& Keller, 2018)

This paper presents the results of a field experiment that was conducted to measure the influence on learning success. Learning success is defined as the increase in knowledge which has taken place and which is influenced by the learning unit and which is measured against the learning objectives defined in the learning unit. This means that a learning success has taken place when at least one or more learning objectives can be achieved by pupils who were not achieved before the unit. 


\section{SCIENTIFIC CONTRIBUTION}

As already mentioned above, Glauser (2018) did not measure the influence of the VR learning unit on learning success. Furthermore, he points out that further, longer-term studies must be carried out in this regard (Glauser \& Keller, 2018). This also implies that such studies must be carried out with larger samples. Glauser also recommends that educational institutions carry out further pilot experiments in order to develop a further understanding of the application (Glauser \& Keller, 2018).

This work can make a concrete contribution here by carrying out the VR course unit micro plastics with a larger sample and comparing it with a control group. Another reason for the investigation with the VR course unit micro plastics in the Swiss secondary school is that the general positive results of digital game-based course units must not be generalized. Hays (2005) explicitly formulates this warning on the grounds that the existing studies give only a fragmented picture in this respect. Hew and Cheung (2010) point out that the results are strongly dependent on sociocultural factors. The implementation of the VR course unit micro plastics in the concrete context of the Swiss secondary level therefore makes sense and leads to new knowledge.

Looking at the literature on the subject of learning in immersive virtual worlds, there is a few other research in this field. If one looks at the literature, it is noticeable that all studies attest to the positive effects of the implementation of VR learning units. All (2016) writes that the positive effects are proven and Mikropulos and Natsis (2011) claim in their conclusion that by the end of the decade (2020) VR technology is mature and suitable for educational applications. Merchant et al. (2014) also come to this conclusion by writing that VR learning units are quoted as "quite effective". At the same time pointing out the various limitations of the studies in the subsequent sentence. However, most of these studies don't deal with immersive VR.

This picture can be seen in all three meta-studies mentioned. The main limitations addressed relate to the type of learning unit, the conduct of the study, the documentation of the procedure and the results. In concrete terms, the following frequently criticized points on the effectiveness of game-based virtual learning units could be identified in the literature:

$>\quad$ Some studies do not contain an exact description of the implementation of the learning unit. It is unclear which variables were used and the statistical information is not adequate (Merchant et al., 2014). Generally insufficient documentation of the procedure and implementation of the course unit. (Clark et al., 2016)

The feedback within the learning unit as well as between the instructor and the trial participant is insufficiently documented and does not allow any conclusions to be drawn about the influence on learning success. (Merchant et al., 2014)

$>\quad$ No control group was implemented for the study or the control group was not exposed to an alternative learning unit (All et al., 2016). The learning unit of the control group was not equivalent in content. (Hays, 2005)

$>\quad$ The methodological approach to the creation of groups and the allocation of learning units was judged insufficient. (All et al., 2016)

$>\quad$ The scientifically correct methodological approach of the study was criticized. For example, there was no pre-test or the post-test was performed too early after the course unit. (All et al., 2016)

$>\quad$ There are no statements in the studies as to how far sustainable learning success has taken place. (Mikropoulos \& Natsis, 2011)

$>$ Missing description of how the relevant influences on learning success between the different groups are kept constant. (All et al., 2016)

At this point the comment of Mikropoulos and Natsis (2011) should be mentioned that there are only a few studies that explicitly document a positive relationship with immersive VR systems. This in turn confirms the above statement that the type of implementation is not always described in detail. Thus, the metastudy by All et. al. (2016) does not reveal whether the VR learning units are fully immersive.

The metastudy by Merchant et al. (2014) is clearer in this regard. It focuses primarily on desktop-based virtual reality systems, i.e. it does not contain any studies with fully immersive systems. Of the 53 studies carried out by Mikropoulos and Natsis (2011), only just 16 concerned fully immersive systems.

Considering the above points of criticism as well as the small number of existing studies explicitly dealing with fully immersive VR systems, a relevant contribution to science can be made within the scope of this work. However, the points of criticism suggest that rigor in methodology must also be given. The rigorousness according to Hevner (2004) describes a methodically correct and scientifically adequate procedure. In order to guarantee rigor, attention must be paid to documenting the procedure and the results as 
specifically as possible, taking the above points of criticism into account.

Under these circumstances, it is understandable that in many studies no concrete statements on the effectiveness of VR learning units can be found. If such statements are available, they are often very vaguely formulated. A good example of this can be found in the work entitled "the effectiveness of instructional games". Hays writes here in his conclusion: "...research has shown that some games can provide effective learning..." (Hays, 2005). As already quoted above, Merchant (2014) writes "quite effective". Mikropoulos (2011) writes in this regard that it is difficult to make statements about the extent to which a learning defect has taken place and the knowledge can later be retrieved and demands that further studies be carried out. Hew and Cheung (2010) go even further here and call for studies that query the learning effect and the available knowledge up to one year after the course unit. Although this time span cannot be covered within this work, a statement on the effectiveness of the VR course unit can be made by implementing a control group and a suitable test setting.

The last point to be mentioned here is the topic of the VR course unit, where a contribution to science can be made. In addition to the small number of immersive VR units, Mikropoulos (2011) also mentions that of the 53 studies only 13 do not come from the fields of mathematics or science. From the later explanations it becomes clear that science refers to topics such as the structure of atoms and the reaction between two atoms. Here, the VR course unit micro plastics (Keller et al., 2019), which takes place in a lake, a laboratory and a barbecue area, certainly offers an exciting change, not only for science, but also for the pupils.

Based on the above mentioned points this work can make a relevant contribution to science. This is especially the case if the rigor of the procedure is guaranteed and the documentation is specific enough.

\section{RESEARCH OBJECTIVE}

For this research work learning with the help of games in virtual worlds is thus defined as a specific subject area. A literature research on this topic shows that this kind of learning leads to promising results. Thus, in his meta-study on the empirical investigation of studies on EVEs, Mikropoulos (2011) concludes that both teachers and pupils are very positive about virtual learning units in education. This statement coincides with the evaluation of the VR course unit micro plastics by Keller (2018), who concludes that all pupils perceived the VR course unit as motivating.

As already mentioned, the subject literature contains many positive statements regarding the influence of VR learning units on learning success. However, many points of criticism can also be identified with regard to the studies conducted. For example, All et al. (2016) point out that in many studies too little methodological precision was applied or that the documentation of the implementation of the learning unit is insufficient. Furthermore, Hays (2005) writes in his study on the effectiveness of game-based learning that the results of such studies cannot be generalized under any circumstances.

For the teachers of the Swiss elementary school, the concrete question is whether VR learning units can lead to positive learning success in their concrete learning environment.

Hypothesis 1: The VR course unit micro plastics (Keller et al., 2019) has a positive influence on the learning success of the pupils.

Hypothesis 2: The VR learning unit micro plastics has a bigger influence on the learning success of the pupils compared to a traditional learning unit based on "paper and pencil"1.

Learning success is defined as the increase in knowledge which has taken place and which is influenced by the learning unit and which is measured against the learning objectives defined in the learning unit. This means that a learning success has taken place when at least one or more learning objectives can be achieved by pupils who were not achieved before the unit.

\section{THE FIELD EXPERIMENT}

The field experiment consists of a pre-test, a post-test, a course unit with VR and a course unit based on a conventional teaching method. Both units have the same learning objectives.

Four classes from a secondary school were available for this field experiment. All four classes are level A, with two in the first year and two in the third (TABLE I). The focus in selecting the classes was on the

${ }^{1}$ https://drive.switch.ch/index.php/s/qCXBcYIJi5ZIMol 
same level. Although the influence of age must be taken into account in the evaluation, the teachers do not consider the age difference to be very influential. In this case, this has to do with the subject matter, as the VR course unit could also be carried out with sixth grade pupils.

Table 1. List of classes and number of pupils

\begin{tabular}{llll}
\hline \hline Class & total & females & males \\
\hline $1 \mathrm{c}$ & 18 & 12 & 6 \\
$1 \mathrm{~d}$ & 20 & 9 & 11 \\
$3 \mathrm{~b}$ & 22 & 15 & 7 \\
$3 \mathrm{c}$ & 22 & 14 & 8 \\
\hline \hline
\end{tabular}

Two types of group allocation are suggested in the literature, random allocation of pupils or classes. The preferred option would be the allocation of pupils, recognizing that this is difficult to achieve in the school and class context. The allocation according to classes is easier, but has several disadvantages. For example, an entire class could be shaped by the teaching style of its class teachers. If an experiment is conducted across several schools, other differences such as city, country, poorer quarter or richer quarter could also have an influence. (All et al., 2016)

Since all test persons for this experiment are from the same school and locality, this factor can be weighted slightly lower. Nevertheless, the attempt was made to take a middle course. For the allocation of the groups, each class was randomly assigned to a group separately for pupils. For example, approximately half of each class absolved the VR course unit and the other half the classical course unit. Technically, this was solved by allocating a random number (according to normal distribution) per pupil. These numbers were then divided into medians and assigned to each half of a group.

In the end, 39 pupils were assigned to the VR course unit and 43 to the classical course unit. The gender distribution is graphically shown in Figure 1 below. On the left the absolute values are shown, on the right the relative values.

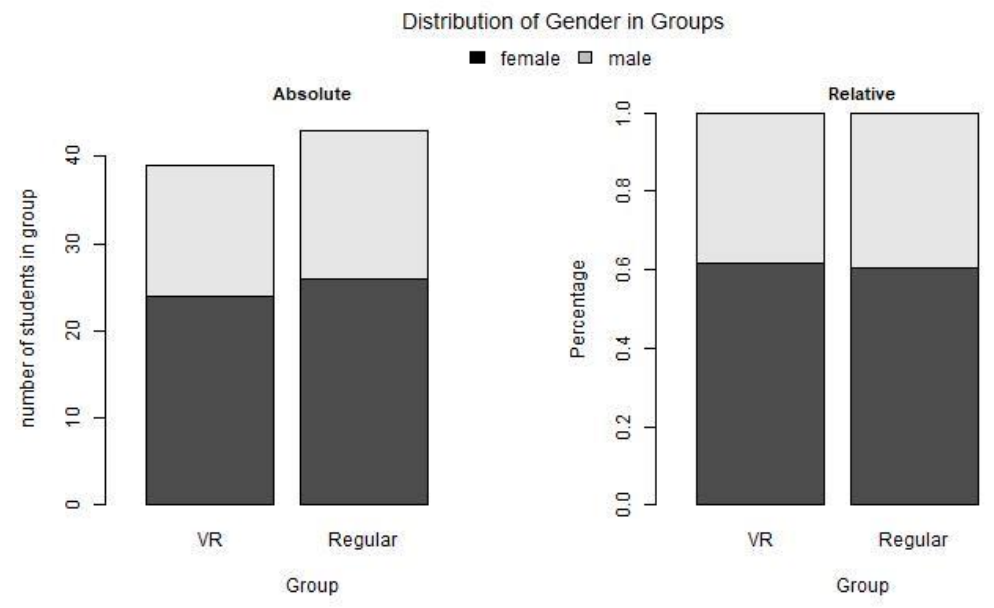

Figure 1. Distribution of gender in groups

\section{RESULTS}

To evaluate the dataset ${ }^{2}$, the results of the pre- and post-tests as well as the information from the individual schedules were converted into tables and imported into the statistical software R-Studio. The application of a statistical test in itself does not yet give any indication as to whether there are significant differences between two samples. For this purpose, a significance level must be determined by the researcher. The significance level can also be described as the probability of error (Kraft \& Landes, 1996). Based on the selected significance level, the $\mathrm{P}$ value of the test is considered. If the $\mathrm{P}$ value is below the selected significance level,

${ }^{2}$ The complet set of raw data including the R-scripts are available at https://drive.switch.ch/index.php/s/ffrZ6Qbj3BTJm6z 
significant differences can be assumed. This means that the differences between the samples examined do not seem to be random (Bortz \& Schuster, 2010). For statistical analyses, the 95\% significance level is often chosen. Stigler argues that this is probably due to Fisher because it can reliably show effect sizes even with small sample sizes. (Stigler, 2008)

For this reason, the $95 \%$ significance level is chosen in each of the following studies in this paper.

\subsection{Overview}

A simple overview of the results of the pre- and post-test is shown in Figure 2. The graph allows a comparison of the results per class and contains the data for both groups.

\section{Comparsion of Test Results}
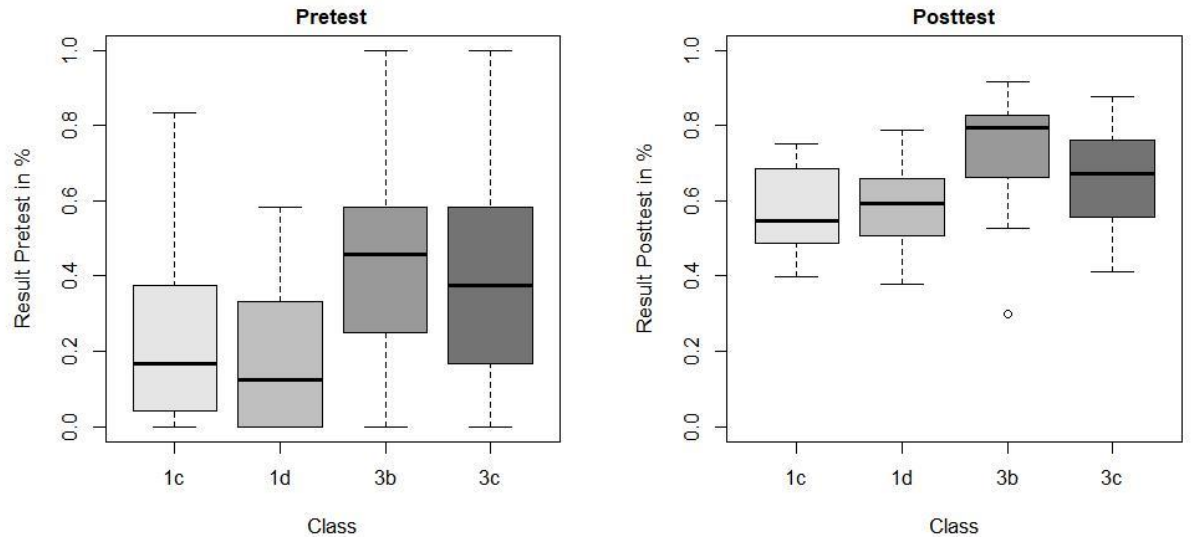

Figure 2. Comparison of test results

If one compares the results of the pre-test and the post-test in Figure 2, it becomes apparent that the results of the pre-test scatter much more strongly than those of the post-test. Furthermore, the medians are visibly lower in the pre-test than in the post-test. It is exciting that both third classes scatter between $0 \%$ and $100 \%$ in the pre-test. This means that some don't know anything, others are already very well informed about the topic of micro plastics. Since nobody reached $100 \%$ in the post-test, this also leads to negative learning successes. This is probably due to the fact that the pre-test and the post-test differ with regard to the questions.

It is also noticeable that the two first and the two third grades each behave similarly. Class $3 b$ seems to have the most knowledge after the post-test. However, this is easy to understand, as the class has the highest median in the pre-test. Otherwise, it can probably be assumed that most pupils have achieved a learning success. The small variance of the post test suggests that both learning units were effective.

\subsection{Hypothesis 1}

The first hypothesis claims that the VR learning unit has a positive influence on learning success. This means that the students have more knowledge about the topic after the VR unit and can retrieve it. The hypothesis can be accepted if the results of the pre- and post-test differ significantly. 

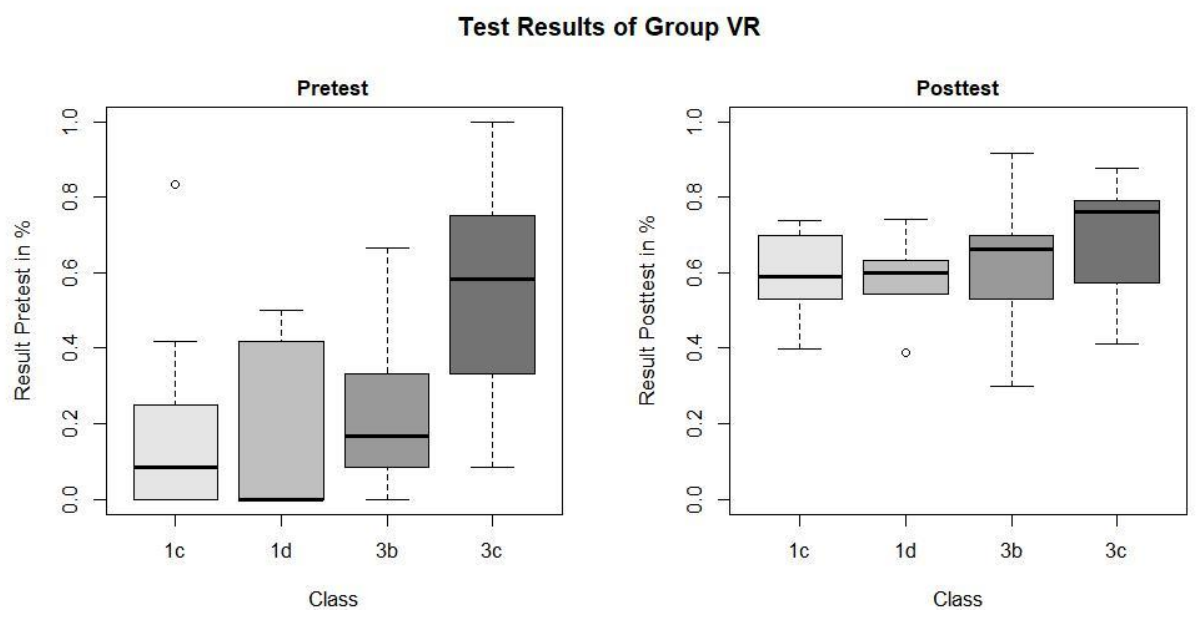

Figure 3. Test result of group VR

The mean value of the results of the post-test is twice as high as in the pre-test. The median of the post-test is even three times higher with half the variance. To compare the results of the pre-test and post-test of the VR learning unit group, these are visualized for each class in Figure 3.

The result of the Wilcox test shows that the results of the pre- and post-test of the VR unit group differ significantly. This can be read from the P-value, which is lower than the defined significance level of 0.05 . Hypothesis 1 can thus be accepted, i.e. the application of a VR learning unit leads to a positive learning success.

\subsection{Hypothesis 2}

The second hypothesis says that a VR learning unit leads to a bigger learning success than a classical learning unit. For the hypothesis to be accepted, the learning success of the VR group must be significantly greater than the learning success of the classical learning unit group. Figure 4 below shows the achieved learning successes (referred to as "gain" in graphics) per group and class as box plots.

\section{Comparsion of Learning Gain}
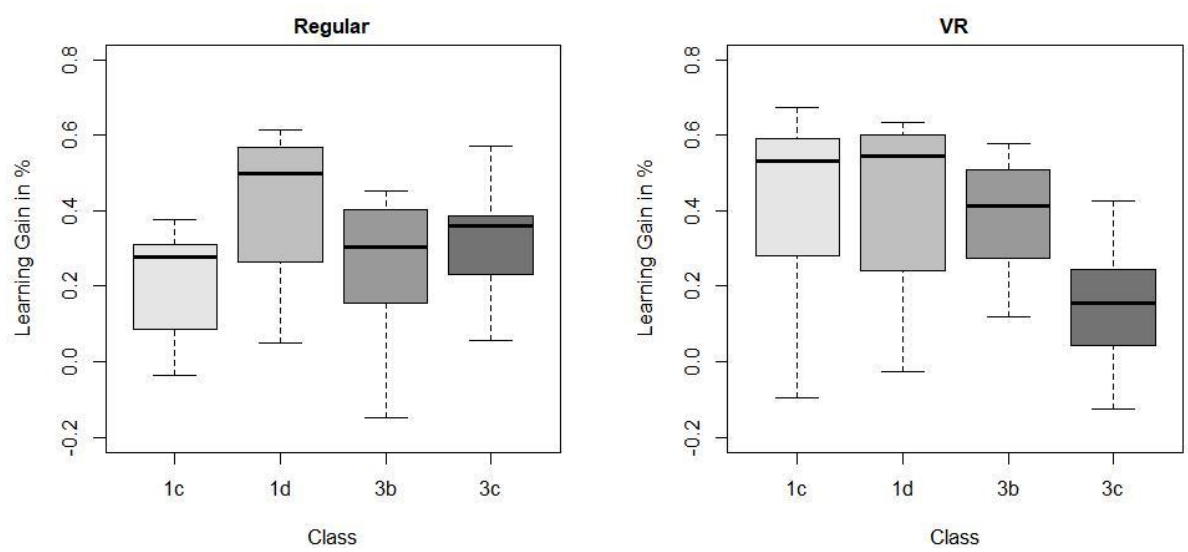

Figure 4. Comparison of learning gain

Looking at Figure 4, it is not clear whether the VR or the traditional learning unit leads to better results. If one compares the learning outcomes between the classes and groups, one sees that the VR unit leads to slightly higher average results, but that the dispersion is also somewhat larger. An exception is class $3 \mathrm{c}$, which has on average completed less well in the VR unit. A possible explanation for the worse result of class $3 \mathrm{c}$ is that class $3 \mathrm{c}$ already achieved the highest average in the pre-test and therefore already had a lot of previous knowledge. 
The average of the VR unit's learning success across all classes is just $2 \%$ higher than that of the regular unit. Conversely, the median is slightly lower. For a concrete statement, however, a statistical test is required. First, the distribution of the learning success must be determined. For this purpose, the data of the learning success is compared with a theoretical normal distribution. A normal distribution can be assumed if the data points shown in Figure 4 follow as closely as possible the straight line in the middle of the grey confidence interval.
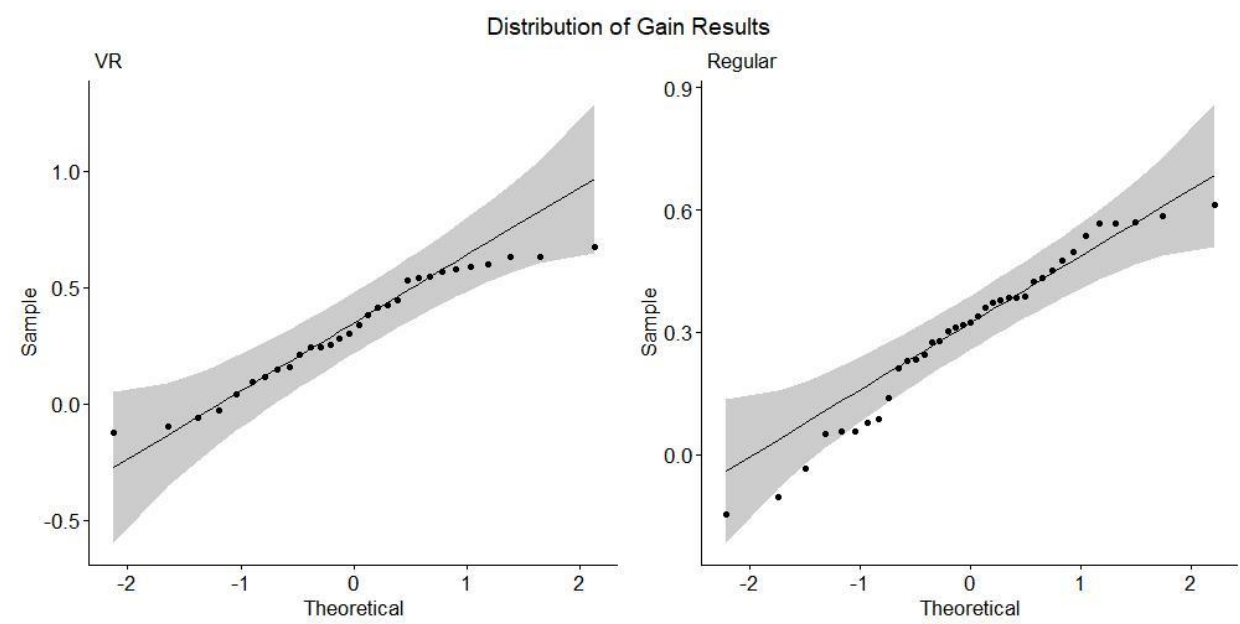

Figure 5. Distribution of gain results

According to Figure 5, a normal distribution can be assumed. However, there are also some data points that are quite far away from the straight line. Another possibility to examine a data set for normal distribution is the Shapiro test. (Bortz \& Schuster, 2010)

The calculation of the $\mathrm{P}$ values by the Shapiro test confirms that a normal distribution can be assumed for the learning success of the VR group as well as for the classical learning unit. This means that the T-test is suitable for testing differences between groups. The Levene test can be used to test the equal distribution of variances (Universität Zürich, 2018). The P-value of the Levene test is greater than 0.05, which means that there are no significant differences between the variances of the learning success of the two groups (Dormann, 2013). Thus, all criteria for the application of the T-test are given.

The calculation of the P-value of the T-test shows a value greater than 0.05 , i.e. there are no significant differences between the learning success of the VR group and the traditional learning unit. It follows that the second hypothesis, that the VR unit is better than the classical unit, cannot be inferred.

\subsection{Further Perceptions}

During the implementation of the VR learning unit it was noticed that there are large differences in how intuitively students move in the VR learning environment. This leads to the question of whether this has an influence on the result, i.e. the learning success. The variable "intuitive behavior" does not exist, but it may be assumed that students who play lots of video games can move more intuitively in a virtual environment. This variable was also collected during the execution and the result of the evaluation is shown in Figure 6. 

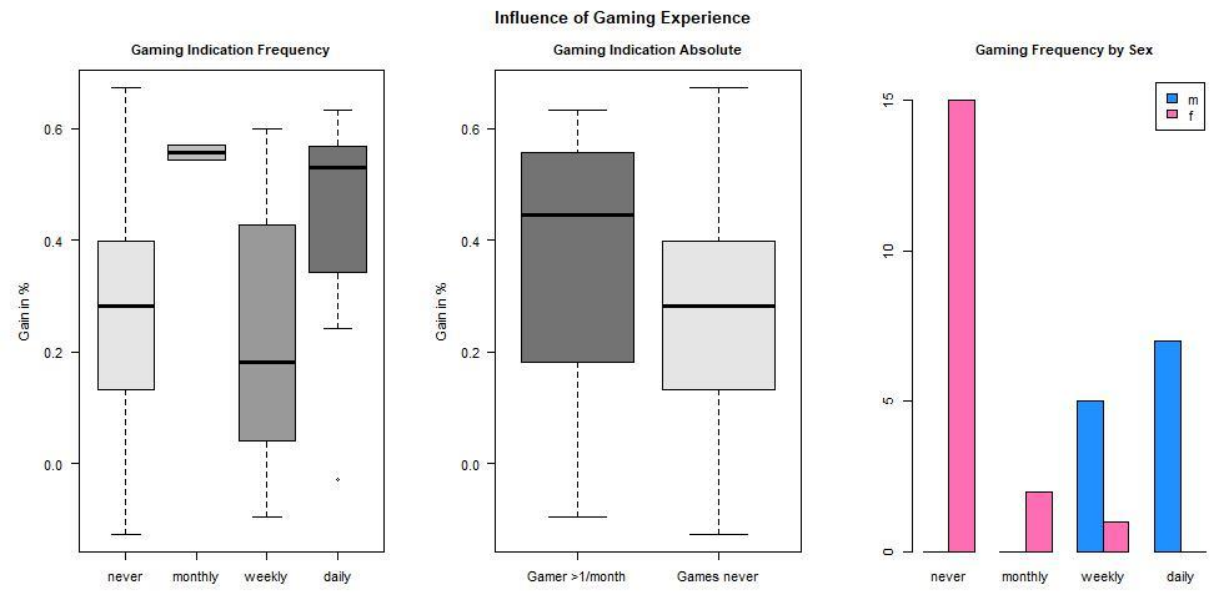

Figure 6. Influence of gaming experience

The middle box plot in Figure 6 shows a similar variance for both groups, but the median is about $15 \%$ higher for groups that play video games at least once a month or more. However, a significant influence of experience with video games cannot be confirmed.

Figure 6 also shows that all pupils have indicated that they play video games at least once a week. For female students, the opposite is true, with the majority saying that they never play video games. This leads to the next question as to whether gender has any influence on the result of the VR group. Figure 7 below shows the learning success and the results of the pre- and post-tests per group.
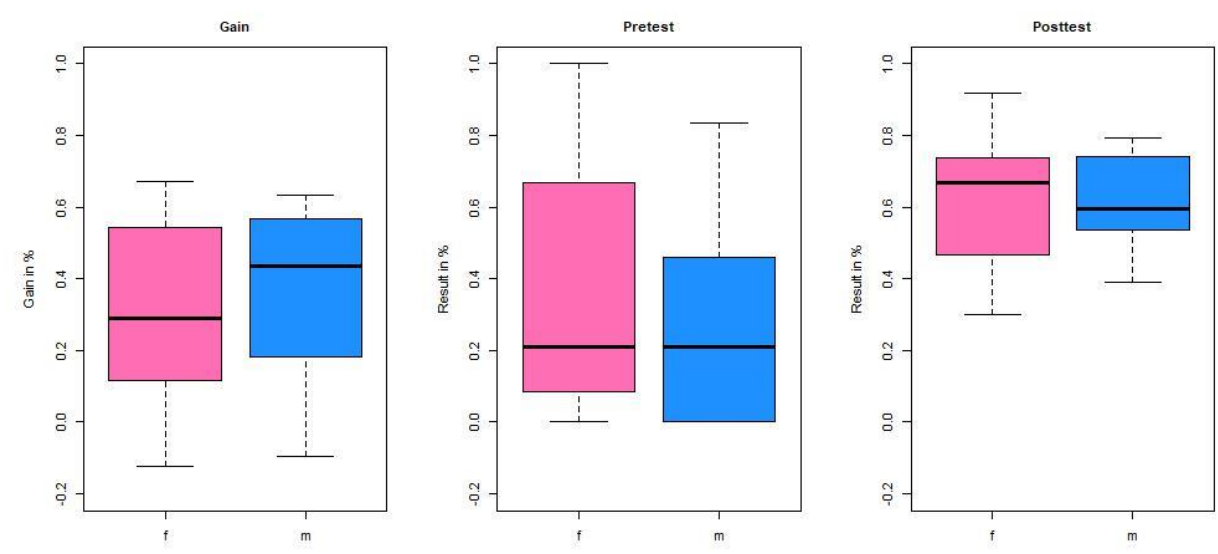

Figure 7. Influence of gender

The male pupils have a median which is about $15 \%$ higher than the one of female pupils. If one also considers the overall results of the pre- and post-tests, it can be seen that the male pupils were slightly worse than the female pupils. In addition, the variance of the male pupils is somewhat lower, which indicates that there are fewer male pupils with very poor learning outcomes than among the female pupils. However, the statistical test shows that gender does not lead to significant differences.

\section{CONCLUSION}

As a result of this work, it can be stated that the research question initially formulated can be confirmed. The implementation of the VR course unit micro plastics has led to a positive learning success. However, no evidence could be found for the assumption that the VR learning unit leads to bigger learning success than with a traditional teaching method in the context of this field experiment. 
The VR unit seems to evoke very different feelings in the pupils. Most of them enjoy the technology and are curious. However, there are also some who either have great reservations beforehand or experience a feeling of fear or discomfort during the learning unit. While this confirms that a VR session has the potential to convey very personal content, it also means that these feelings may have a negative impact on the learning success of individual pupils. This also includes the observed factor of intuition, i.e. how easy or difficult it was for individual pupils to navigate within the VR unit. These factors were not collected in the context of this field experiment, which is why further statements in this respect are not possible. A further confirmation for this influence could be the larger scatter of the VR learning unit compared to the traditional learning unit. This would explain that there are greater differences in learning success within the VR group.

In this field experiment the learning unit had to be abandoned by four female students. Hence, the learning content could not be conveyed and furthermore the pupils left the learning unit with negative feelings. It is assumed that this effect will diminish with the future pervasion of the technology. A VR learning unit would become something "normal" and "known".

\section{REFERENCES}

All, A., Nuñez Castellar, E. P., \& Van Looy, J. (2016). Assessing the effectiveness of digital game-based learning: Best practices. Computers \& Education, 92-93, 90-103. https://doi.org/10.1016/j.compedu.2015.10.007

Bortz, J., \& Schuster, C. (2010). Statistik für Human- und Sozialwissenschaftler (7., vollständig überarbeitete und erweiterte Auflage). Springer.

Clark, D. B., Tanner-Smith, E. E., \& Killingsworth, S. S. (2016). Digital Games, Design, and Learning: A Systematic Review and Meta-Analysis. Review of Educational Research, 86(1), $79-122$. https://doi.org/10.3102/0034654315582065

Dormann, C. F. (2013). Das Lineare Modell: T-Test und ANOVA in R. In C. F. Dormann, Parametrische Statistik (pp. 209-223). Springer Berlin Heidelberg. https://doi.org/10.1007/978-3-642-34786-3_12

Glauser, P., \& Keller, T. (2018). Einsatz von Virtual Reality an der Schweizer Volksschule. https://drive.switch.ch/index.php/s/jnO5w4a8aozjlKt

Hays, R. T. (2005). The Effectiveness of Instructional Games: A Literature Review and Discussion: Defense Technical Information Center. https://doi.org/10.21236/ADA441935

Hevner, A. R., March, S. T., Park, J., \& Ram, S. (2004). Design Science in Information Systems Research. 32.

Hew, K. F., \& Cheung, W. S. (2010). Use of three-dimensional (3-D) immersive virtual worlds in K-12 and higher education settings: A review of the research: Use of 3-D in K-12 and higher education. British Journal of Educational Technology, 41(1), 33-55. https://doi.org/10.1111/j.1467-8535.2008.00900.x

Keller, T., Glauser, P., \& Brucker-Kley, E. (2018). Virtual Reality at secondary schools: First resulsts. 53-60. http://www.iadisportal.org/digital-library/virtual-reality-at-secondary-school-\%C2\%96-first-results

Keller, T., Hebeisen, A., Glauser, P., \& Turnheer, L. (2019). Neue Lehrkonzepte an Schulen - Einsatz von Virtual Reality. Neue Lehrkonzepte. https://neuelehrkonzepte.ch/

Kraft, M., \& Landes, T. R. (1996). Statistische Methoden: Eine Einführung für das Grundstudium in den Wirtschaftsund Sozialwissenschaften (3., durchges. und aktualisierte Aufl). Physica-Verl.

Merchant, Z., Goetz, E. T., Cifuentes, L., Keeney-Kennicutt, W., \& Davis, T. J. (2014). Effectiveness of virtual realitybased instruction on students' learning outcomes in K-12 and higher education: A meta-analysis. Computers \& Education, 70, 29-40. https://doi.org/10.1016/j.compedu.2013.07.033

Mikropoulos, T. A., \& Natsis, A. (2011). Educational virtual environments: A ten-year review of empirical research (1999-2009). Computers \& Education, 56(3), 769-780. https://doi.org/10.1016/j.compedu.2010.10.020

Stigler, S. (2008). Fisher and the 5\% Level. CHANCE, 21(4), 12-12. https://doi.org/10.1080/09332480.2008.10722926

Universität Zürich. (2018, August 13). Methodenberatung Universität Zürich-Wilcoxon Test. Wilcoxon-Test. https://www.methodenberatung.uzh.ch/de/datenanalyse_spss/unterschiede/zentral/wilkoxon.html 